\title{
Aplicabilidade do Índice SOFA em pacientes com Sepse em uma unidade de terapia intensiva de um hospital na cidade de Fortaleza/CE
}

\author{
Applicability of SOFA Index in Patients With Sepsis in an Intensive Care \\ Unit of a Hospital in The City Of Fortaleza/CE
}

Hassan Kelvin Feitosa EvanGELISTA

Centro Universitário Christus, Fortaleza/CE - Brasil

Joselito de Oliveira Neto Faculdade Maurício de Nassau, Fortaleza-CE - Brasil Íris Pereira Magalhães Centro Universitário Christus. Fortaleza/CE - Brasil

Raquel Horn Vasconcelos de Oliveira

Universidade de Fortaleza. Fortale$\mathrm{za} / \mathrm{CE}$ - Brasil

Nathalie Ommundsen Pessoa Faculdade de Tecnologia Intensiva, Fortaleza/CE - Brasil

Márcia Cardinalle Correia

VIANA

Centro Universitário Christus.

Fortaleza/CE - Brasil
Resumo O índice SOFA tem na sua aplicação a avaliação das disfunções orgânicas, e a sepse como um agravante nas Unidades de Terapia Intensiva (UTI's), aumentando os episódios de falências orgânicas. Avaliar essa aplicabilidade pode ser de grande relevância, tratando-se de uma equipe interdisciplinar que constantemente necessita de métodos auxiliares. Foram analisados 20 pacientes com sepse, admitidos na UTI de um hospital público do município de Fortaleza/CE, sendo coletados dados a partir de prontuários por meio de um estudo prospectivo, observacional do tipo censo entre novembro de 2011 a abril de 2012. A taxa de mortalidade global foi de $55 \%$, a maioria dos pacientes não apresentou comorbidades e o sistema cardiovascular foi o mais afetado quanto à disfunção orgânica. Como um recurso auxiliar em UTI, o índice SOFA torna-se possível a aplicação para aperfeiçoar a qualidade da avaliação e o acompanhamento junto ao paciente com sepse, e assim possibilitando que as disfunções orgânicas sejam controladas facilmente.

Palavras-chave: Sepse. SOFA. Hospitalização.

Abstract The SOFA index has in its application the evaluation of the organic dysfunctions, and sepsis as an aggravating factor in the Intensive Care Units (ICUs), increasing the episodes of organic failures. Evaluating this applicability can be of great relevance, being an interdisciplinary team that constantly needs ancillary methods. Twenty patients with sepsis admitted to the ICU of a public hospital in the city of Fortaleza/ CE were analyzed, and data were collected from medical records through a prospective, observational, census-type study between November 2011 and April 2012. The overall mortality was 55\%, most patients had no comorbidities, and the cardiovascular system was the most affected in terms of organ dysfunction. As an auxiliary resource in the ICU, the SOFA index makes possible the application to improve the quality of the evaluation and the accompaniment to the patient with sepsis, thus allowing the organic dysfunctions to be controlled easily.

Key-words: Sepsis. COUCH. Hospitalization. 


\section{INTRODUÇÃO}

Atualmente, as Unidades de Terapia Intensiva (UTI's) são as unidades mais críticas nos ambientes hospitalares, e priorizam o atendimento ao paciente com provável comprometimento das funções vitais devido à falha de um ou mais sistemas orgânicos consequentes a doenças, traumatismos ou intoxicações. Com incertezas no tocante à sobrevivência e ao aparecimento de eventos catastróficos, é considerada como uma unidade de elevada tensão, o que produz ansiedade, facilmente traduzida em estresse. A verdadeira meta da terapia intensiva é a redução da morbimortalidade. ${ }^{1,2}$

Muitos desses pacientes podem evoluir com infecção importante e desenvolver sepse. O desenvolvimento da sepse depende das relações estabelecidas entre o microrganismo e o hospedeiro destacando-se que, muitos dos elementos relativos ao seu aparecimento permanecem obscuros, provavelmente pela falta de uma compreensão mais adequada no que se refere à imunidade, inflamação e coagulação. ${ }^{3,4,5,6}$

Os pacientes sépticos exigem maior tempo de internação na UTI, resultando num maior custo de tratamento comparado a outros pacientes. Nos EUA, a sepse é a $10^{a}$ causa de morte, sendo apontado por um estudo de fármaco-economia europeu um gasto direto de 23.000 a 34.000 euros. ${ }^{7,8}$

Criado em 1995 e originalmente titulado do inglês de Sepsis-related Organ Failure Assessment (Avaliação Sequencial da Falência Orgânica-SOFA), esse índice prognóstico foi feito para a avaliação de morbidades em pacientes sépticos. Analisa seis sistemas orgânicos graduando entre 0 e 4 pontos de acordo com o grau de disfunção orgânica/ falência, assim como avaliar a eficácia das diversas terapêuticas utilizadas. ${ }^{9,10,11}$
Para o diagnóstico e a classificação dos estágios da sepse, seguiram-se as orientações da Conferência de Consenso de 1992 pelo American College of Chest Physicians e da Society of Critical Care Medicine. ${ }^{12}$

A sepse foi definida como um processo infeccioso associado a dois ou mais critérios da síndrome da resposta inflamatória sistêmica: temperatura $\geq 38^{\circ} \mathrm{C}$ ou $\leq 36^{\circ} \mathrm{C}$; frequência cardíaca $\geq 90 \mathrm{bpm}$; frequência respiratória $\geq 20 \mathrm{mov} / \mathrm{min}$, ou $\mathrm{PaCO}_{2} \leq 32$ $\mathrm{mmHg}$, ou necessidade de ventilação mecânica; leucócitos $\geq 12.000 \mathrm{cél} / \mathrm{mm}^{3}$, ou < 4.000 cél $/ \mathrm{mm}^{3}$, ou $10 \%$ de células imaturas. ${ }^{12}$

A sepse grave, além dos critérios descritos, apresentava ao menos uma falência orgânica e choque séptico, os pacientes, além de preencherem os critérios de sepse, tinham pressão sistólica $\leq 90 \mathrm{mmHg}$ após reposição volêmica, com necessidade de drogas vasoativas. ${ }^{12}$

O objetivo deste estudo foi verificar a aplicabilidade do índice SOFA em pacientes com sepse em uma UTI de um hospital na cidade de Fortaleza/CE.

\section{MÉtodos}

Trata-se de um estudo de campo do tipo documental e prospectivo, em uma UTI geral da rede pública do município de Fortale$\mathrm{za} / \mathrm{CE}$ composta de 12 leitos.

A pesquisa foi aprovada pelo Comitê de Ética e Pesquisa da unidade pesquisada (protocolo $\mathrm{N}^{\circ}$. 560/2011). O chefe da unidade assinou o Termo de Consentimento Informado para a coleta de dados dos prontuários.

Para a realização desta pesquisa, foram obedecidos todos os preceitos éticos de pesquisas em seres humanos que regem da confidencialidade, sigilo, anonimato, autonomia, beneficência, não maleficência, justiça e equidade, regulamentados pela 
resolução 196/96 do Conselho Nacional de Saúde/Ministério da Saúde. ${ }^{13}$

A população constou de pacientes que estavam internados no período de novembro de 2011 a abril de 2012 e evoluíram com diagnóstico de sepse, sepse grave ou choque séptico. Cada paciente foi acompanhado até o $28^{\circ}$. dia ou até a sua alta da UTI ou óbito.

Foram excluídos os pacientes que retornaram à UTI, com novo episódio de sepse, após já terem participado do estudo na internação anterior.
A coleta de dados clínicos e laboratoriais foi realizada pelos pesquisadores por meio de exame do paciente e análise da história clínica registrada em prontuário. Os testes laboratoriais foram feitos pelo próprio laboratório do hospital. Após o diagnóstico e a classificação da sepse, o índice SOFA (Tabela 1) foi medido no $1^{\circ}$., $3^{\circ} ., 5^{\circ} ., 7^{\circ} ., 14^{\circ} ., 28^{\circ}$. dias após a inclusão do paciente no estudo. Para a definição do desfecho clínico, obteve-se a alta ou óbito na UTI.

\section{TABELA 1: Índice SOFA}

\begin{tabular}{|c|c|c|c|c|c|}
\hline SOFA Escore & 0 & 1 & 2 & 3 & 4 \\
\hline \multicolumn{6}{|l|}{ Respiração } \\
\hline $\mathrm{PaO} 2$ / FiO2 (a) & $>400$ & $<400$ & $<300$ & $<200$ (a) & $<10$ \\
\hline \multicolumn{6}{|l|}{ Coagulação } \\
\hline Plaquetas $10^{3} / \mathrm{mm}^{3}$ & $>150$ & $<150$ & $<100$ & $<50$ & $<20$ \\
\hline \multicolumn{6}{|l|}{ Hipotensão } \\
\hline Cardiovascular (b) & $\mathrm{PAM}>70$ & $\mathrm{PAM}<70$ & Dopamina $\leq 5$ & Dopamina $>5$ & \\
\hline \multicolumn{6}{|l|}{ Dopamina $>15$} \\
\hline & & & ou Dobutamina, & ou Epinefrina $\leq 0.1$ & ou \\
\hline \multicolumn{6}{|l|}{ Epinefrina $>0.1$} \\
\hline & & & qualquer dose & ou Norepinefrina $\leq 0.1$ & ou \\
\hline \multicolumn{6}{|l|}{ Norepinefrina $>0.1$} \\
\hline \multicolumn{6}{|l|}{ Fígado } \\
\hline Bilirrubina mg/dl & $<1.2$ & $1.2-1.9$ & $2.0-5.9$ & $6.0-11.9$ & $>12.0$ \\
\hline \multicolumn{6}{|c|}{ SNC escala de coma } \\
\hline de Glasgow & $>14$ & $13-14$ & $10-12$ & $6-9$ & $<6$ \\
\hline \multicolumn{6}{|l|}{ Renal creatinina } \\
\hline ou débito urinário & $<1.2$ & $1.2-1.9$ & $2.0-3.4$ & $3.5-4.9<500$ & \\
\hline$>5$ ou $<200$ & & & & & \\
\hline
\end{tabular}

(a) Com suporte ventilatório (b) Agentes adrenérgicos administrados por pelo menos 1 hora (doses em g/Kg/min) Fonte: Vincent (1996). 
Os dados coletados foram tabulados com o auxílio do Microsoft Office Excel versão 2002 e posteriormente analisados pelo Software estatístico SPSS (Statistical Package for the Social Sciences) versão 16.0, por meio de análises descritivas, aplicando valores de médias e determinando as frequências das complicações, alta e óbito.

Foram também utilizados o teste $\mathrm{t}$ -student para comparar valores do índice SOFA entre os dias que houve medição, e o teste Qui-quadrado para observar a relação entre o índice e as complicações orgânicas, percebendo um valor significante $\mathrm{p} \leq 0,05$. Os resultados foram expressos em forma de tabelas e gráficos para melhor compressão.

\section{RESUlTADOS E DISCUSSÃo}

De novembro de 2011 a março de 2012 foram admitidos 99 pacientes na UTI, onde 20 deles evoluíram com sepse. Desses pacientes estudados, 12 (60\%) eram do sexo masculino e oito (40\%) do sexo feminino, com a idade entre 20 a 85 anos com média de 64,1 anos. Os motivos para a internação estiveram relacionados à causa cirúrgica (50\%), clínica (45\%) e obstétrica (5\%). A taxa de mortalidade global foi de $55 \%$.

Dos 20 pacientes, apenas quatro possuíam alguma cardiopatia, não tendo qualquer valor estatístico relevante. Observou-se que $13(65 \%)$ não apresentaram qualquer comorbidade, e entre eles, nove evoluíram ao óbito e quatro tiveram alta para a enfermaria.

Na Figura 1 estão descritos alguns dos principais órgãos afetados com disfunção. Escores com valores $\geq$ a 3 para cada órgão, indicam uma disfunção orgânica.

Figura 1: Quantitativo de pacientes com acometimento de sistemas/órgão de acordo com índice $S O F A \geq 3$.

\begin{tabular}{|c|c|c|c|c|c|c|}
\hline & $1^{\circ}$. dia & $3^{\circ}$. dia & $5^{\circ}$. dia & $7^{\circ}$. dia & $14^{\circ}$. dia & $28^{\circ} . \mathrm{dic}$ \\
\hline Respiratório & 5 & 4 & 3 & 2 & 3 & 0 \\
\hline Coagulação & 2 & 1 & 2 & 1 & 0 & 0 \\
\hline Fígado & 1 & 1 & 1 & 1 & 0 & 0 \\
\hline Cardiovascular & 11 & 12 & 11 & 6 & 5 & 2 \\
\hline Neurológico & 4 & 4 & 3 & 2 & 2 & 0 \\
\hline Renal & 3 & 4 & 4 & 5 & 2 & 2 \\
\hline
\end{tabular}

Na Tabela 2 encontra-se a análise comparativa entre os dias avaliados pelo índice SOFA. Observa-se significância estatística entre o $1^{\circ}$. e o $3^{\circ}$. dia de coleta, com um valor significativo no sistema coagulação/plaquetas, onde três pacientes apresentaram valor de pontuação do índice SOFA maior ou igual a 3. 
TABELA 2: Análise do Índice SOFA de acordo com os dias coletados (1º., $3^{\mathbf{0}}$., $5^{\mathbf{0}}$., $7^{\mathbf{0}}$., $14^{\mathbf{o}}$. e $28^{\mathbf{0}}$.).

\begin{tabular}{|c|c|c|c|c|c|}
\hline Sistemas/órgão & $\begin{array}{c}\text { Valor } \mathrm{p} \\
1^{\circ} \text {. e } 3^{\circ} \text {. dias }\end{array}$ & $\begin{array}{l}\text { Valor p } \\
1^{\circ} \text {. e } 5^{\mathbf{o}} \text {. } \\
\text { dias }\end{array}$ & $\begin{array}{c}\text { Valor } \mathrm{p} \\
1^{\text {o. }} \text { e } 7^{\mathbf{o}} \text {. dias }\end{array}$ & $\begin{array}{l}\text { Valor p } \\
\begin{array}{c}1^{\circ} . \text { e } 14^{\circ} . \\
\text { dias }\end{array}\end{array}$ & $\begin{array}{c}\text { Valor } \mathrm{p} \\
1^{\circ} \text {. e } 28^{\circ} \text {. dias }\end{array}$ \\
\hline Sistema Respiratório & 0,66 & 0,38 & 0,86 & 0,85 & 0,46 \\
\hline Coagulação/Plaquetas & 0,05 & 0,15 & 0,27 & 1 & - \\
\hline Fígado & - & - & - & - & - \\
\hline Sistema Cardiovascular & 1 & 0,78 & 0,20 & 0,74 & 0,61 \\
\hline Sistema Neurológico & 0,17 & 0,42 & - & - & - \\
\hline Sistema Renal & 1 & 0,49 & 1 & 0,72 & 0,77 \\
\hline
\end{tabular}

Na sepse, ocorre uma resposta inflamatória aguda ao insulto infeccioso. Algumas proteínas liberadas, como as citocinas, podem apresentar efeito autócrino (quando a célula-alvo é a própria célula que secreta a citocina), efeito parácrino (quando a célulaalvo é vizinha) ou efeito endócrino promovendo reações fora dos limites inflamatórios localizados e resultando em manifestações sistêmicas. A interação complexa entre citocinas e moléculas neutralizadoras de citocinas provavelmente determina a apresentação clínica e o curso da sepse. ${ }^{14,15}$

Alguns dados a respeito das características dos pacientes com sepse nas UTI's do Nordeste estão escassos. O estudo BASES forneceu informações epidemiológicas muito interessante sobre a sepse nas UTI's do Sul e Sudeste do país. No entanto, o Brasil é um país de dimensões continentais e com uma população heterogênea sendo necessário que cada região ou serviço de saúde saiba o real perfil epidemiológico dos pacientes com sepses, para definir prioridades de intervenção com a intenção de melhorar 0 atendimento a esse grupo de pacientes. ${ }^{16,8}$

O desenvolvimento de vários sistemas de escore fornece ao profissional intensi- vista a habilidade de acuradamente aferir a gravidade da disfunção orgânica. A principal diferença entre o índice SOFA e outros escores desenvolvidos é a utilização de dados clínicos e laboratoriais de fácil aquisição, e a definição mais objetiva da disfunção cardiovascular, tornando-o atraente como sistema de análise de disfunção orgânica. ${ }^{17}$

O índice SOFA foi concebido não para prever o resultado, mas para descrever uma sequência de complicações no paciente crítico, muito embora qualquer avaliação de morbidade deva ser relacionada com a mortalidade para alguns graus. ${ }^{18}$

O tempo de evolução é um fator fundamental por várias razões. Pacientes que morrem muito cedo não têm tempo para desenvolver uma relação disfunção/falência. O tempo de evolução auxilia em um melhor entendimento do processo infeccioso da sepse como um processo natural ou em influência de intervenções terapêuticas. Por esse e outros motivos a coleta diária deve ser seguida. ${ }^{19}$

Neste estudo, o SOFA foi associado à mortalidade, mostrando que quanto mais grave for a forma de apresentação inicial da doença, tanto maior a mortalidade, revelan- 
do uma maior probabilidade de idosos irem a óbito. Confirmando o conceito atual de que a sepse é uma doença dependente do tempo. ${ }^{10}$

Divergente aos resultados encontrados no presente estudo que obteve taxa de mortalidade de $55 \%$, outros trabalhos obtiveram taxa global de mortalidade em UTI entre $21,8 \%$ e $46,4 \% .{ }^{20,8}$ Esse resultado aponta a necessidade de mais aprimoramentos para a prevenção e o tratamento das sepses hospitalares, o que reforça a importância da utilização do índice SOFA nessa condição clínica.

Os índices de prognóstico devem ser utilizados na Medicina Intensiva atual para descrever o grau de disfunção orgânica de forma quantitativa, transformando a gravidade da doença do paciente em valor numérico, por meio de dados clínicos, laboratoriais e por vezes, pelo tipo de intervenções utilizadas. Além disso, seu valor está em predizer e agregar o impacto da gravidade da doença sobre a morbidade e a mortalidade dos pacientes. ${ }^{21}$

Neste estudo, a relação entre o primeiro e o terceiro dia de coleta $(\mathrm{p}=0,05)$, revelou um pior desfecho de internação. Isso pode significar que os pacientes permanecem grave apesar da terapêutica instituída no período inicial.

A partir dos dados obtidos, observou-se que as disfunções cardiovasculares e respiratórias foram as mais comumente encontradas. Entretanto, as disfunções neurológicas e cardiovasculares estiveram associadas tanto de forma isolada como combinada o maior risco de morte, achados que vão de encontro com aqueles já registrados na literatura. ${ }^{18,17}$

No presente estudo, as disfunções ou falências orgânicas mostraram uma maior prevalência do sistema cardiovascular. Pa- cientes infectados apresentam um consistente aumento no grau de disfunção orgânica, confirmando a ampla correlação entre sepse e falência orgânica múltipla, entre eles, o coração. ${ }^{10,22}$

Apontamos como limite neste estudo, a falta de resultados de exames relacionados ao sistema hepático. Também foi pouco registrada a pontuação do Glasgow. Essa falta de dados pode comprometer a avaliação das disfunções desses órgãos e causar alterações significativas.

\section{CONSIDERAÇões Finais}

Os resultados deste estudo confirmam o índice SOFA como um ótimo sistema de avaliação da disfunção orgânica. É um importante indicador prognóstico, podendo ser utilizado para quantificar o impacto de intervenções terapêuticas sobre a morbidade, bem como, na identificação de grupos de maior risco e que merecerão maior monitorização.

Prima-se ainda a necessidade de uma equipe multidisciplinar (médicos, enfermeiros, fisioterapeutas, auxiliares de enfermagem) capacitada para abordar esses pacientes e empenhada em elaborar protocolos de tratamento que tornem essas abordagens cada vez mais efetivas.

A avaliação da disfunção orgânica permanece difícil, mas acreditamos que o desenvolvimento da pontuação SOFA representa uma abordagem valiosa.

Os critérios usados e especialmente os valores individuais para cada um dos parâmetros utilizados no índice não devem ser considerados como definitivos, mas podem ser alterados quando os dados são suficientemente coletados. 
O índice SOFA, como um recurso auxiliar em UTI, torna-se possível na aplicação para aperfeiçoar a qualidade da avaliação e acompanhamento junto ao paciente com sepse, acompanhando a disfunção orgânica, se presente, e assim ocasionando a diminui- ção das comorbidades e mortalidade, mas facilitando a alta para a enfermaria.

\section{Agradecimentos}

Ao chefe da UTI pela autorização, apoio e incentivo à pesquisa.

\section{REFERÊNCIAS}

1. Friedman G., Victorino JA. In: Sepse. Rotinas em Terapia Intensiva, 3. ed. Porto Alegre: Artmed, 2003.

2. Baasch D., Laner AS. Os significados do trabalho em unidades de terapia intensiva de dois hospitais brasileiros. Ciência \& Saúde Coletiva, 2011; 16 (1): 1097-5.

3. Pereira Júnior GA et al. Fisiopatologia da sepse e suas implicações terapêuticas. Simpósio: medicina intensiva: I. infecção e choque, jul./set. 1998; 31, cap. II: 349-62.

4. Bone RC et al. Definitions for sepsis and organ failure and guidelines for the use of innovative therapies in sepsis. The ACCP/SCCM Consensus Conference Committee. American College of Chest Physicians/Society of Critical Care Medicine. Chest, 1992; 101: 1644-55.

5. Siqueira-Batista R et al. Sepse: atualidades e perspectivas. Revista Brasileira de Anestesiologia, jul./mar. 2011; 23 (2): 207-16.

6. Campos EV et al. Uso do MODS Modificado em Pacientes Sépticos no Departamento de Emergência para Predizer Mortalidade. Revista Brasileira de Terapia Intensiva, abr./jun. 2005; 17 (1): 74-9.

7. Koenig A. et al. Estimativa do impacto econômico da implantação de um protocolo hospitalar para detecção e tratamento precoce de sepse grave em hospitais públicos e privados do sul do Brasil. Revista Brasileira de Terapia Intensiva. Santa Catarina, 2010; 22 (3): 213-19.

8. Koury JCA, Lacerda HR, Barros Neto AJ. Características da população com sepse em unidade de terapia intensiva de um hospital terciário e privado da cidade do recife. Revista Brasileira de Terapia Intensiva, jan./mar. 2006; 18 (1): 52-8.

9. Silva E., Pinheiro C., Michels Júnior V. Epidemiologia. Revista Brasileira de Terapia Intensiva, abr./jun. 2004; 16 (2): 97-01.

10. Lemos RLL et al. Associação do SOFA com a mortalidade de idosos com sepse grave e choque séptico. Revista Brasileira de Terapia Intensiva, out./dez. 2005; 17 (4): 246-50.

11. Silva E., Otero JB. Disfunção de Múltiplos Órgãos. Revista Brasileira de Terapia Intensiva. São Paulo, abr./jun. 2004; 16 (2): 114-18.

12. American College of Chest Physicians/Society of Critical Care Medicine Consensus Conference: definitions for sepsis and organ failure and guidelines for the use of innovative therapies in sepsis. Crit Care Med, 1992.

13. BRASIL. Resolução CSN nº. 196, de 10 de outubro de 1996. Aprova diretrizes e normas regulamentadoras de pesquisas envolvendo seres humanos. Diário Oficial da União, Brasília, DF, n. 201,16 out. 1996. Seção 1, p. 21082.

14. Miranda FG. Sepse. Jornal Brasileiro de Medicina, ago. 2007; 93 (2).

15. Paiva RA, David CM, Domont GB. Proteômica na sepse: estudo piloto. Revista Brasileira de Terapia Intensiva. Rio de Janeiro, 2010; 22 (4): 403-12. 
16. Sales Junior JAL et al. Sepse Brasil: estudo epidemiológico da sepse em unidades de terapia intensiva brasileiras. Revista Brasileira de Terapia Intensiva, jan./mar. 2006; 18 (1): 9-17.

17. Sampaio FBA et al. Utilização do SOFA ESCORE na Avaliação da Incidência de Disfunção Orgânica em Pacientes Portadores de Patologia Cardiovascular. Revista SOCERJ. mar./abr. 2005, 18 (2).

18. Vincent JL, Moreno R., Takala J., Willats S., De Mendonça A., Bruining H., et al. The SOFA (sepsis - related organ failure assessment) score to describe organ dysfunction / failure. On behalf of the Working Group on Sepsis - Related Problems of the European Society of Intensive Care Medicine. Intens Care Med. 1996.

19. Machado FR et al. Associação entre a disfunção orgânica e as concentrações de citocinas na fase inicial do choque séptico. Revista Brasileira de Terapia Intensiva. São Paulo, 2011, 23 (4): 426-33.

20. Zanon F. et al. Sepse na unidade de terapia intensiva: Etiologias, Fatores prognósticos e Mortalidade. Revista Brasileira de Terapia Intensiva. Rio Grande do Sul, abr./jun. 2008, 20 (2).

21. Bueno LO et al. Avaliação dos índices prognósticos SOFA e MODS em pacientes após parada cardiorrespiratória em unidade de terapia intensiva geral. Revista Brasileira de Terapia Intensiva, jul./set. 2005, 17 (3): 162-64.

22. Boechat AL, Boechat NO. Sepse: Diagnóstico e tratamento. Revista Brasileira de Clínica Médica. São Paulo, set./out. 2010, 8 (5): 420-27.

23.

24. Dados dos autores:

25. Hassan Kelvin Feitosa Evangelista

26. Curso de Fisioterapia. Centro Universitário Christus, Fortaleza/CE - Brasil. hassankelvin@hotmail.com

27. Joselito de Oliveira Neto

28. Fisioterapeuta. Doutorando do Programa de Pós-graduação em Biotecnologia da Universidade Federal do Ceará - UFC. Docente do Curso de Fisioterapia da Faculdade Maurício de Nassau, Fortaleza-CE - Brasil. joselitoneto@yahoo.com.br

29. Íris Pereira Magalhães

30. Curso de Fisioterapia, Centro Universitário Christus. Fortaleza/CE - Brasil. iris.pereira@hotmail.com

31. Raquel Horn Vasconcelos de Oliveira

32. Fonoaudióloga, Universidade de Fortaleza. Fortaleza/CE - Brasil. raquel_hv82@hotmail.com

33. Nathalie Ommundsen Pessoa

34. Docente do Curso de Biomedicina, Faculdade de Tecnologia Intensiva, Fortaleza/CE - Brasil. nathalieop@gmail.com

35. Márcia Cardinalle Correia Viana

36. Fisioterapeuta. Mestre em Saúde Pública pela Universidade Estadual do Ceará. Docente do Curso de Fisioterapia, Centro Universitário Christus. Fortaleza/CE - Brasil. marcia.cardinalle@ hotmail.com

Submetido em: 06/05/2017

Aceito em: 30/01/2018 